\title{
Epigenetic Targeted Therapy for Diffuse Intrinsic Pontine Glioma
}

\author{
Rintaro HASHIZUME ${ }^{1,2}$ \\ ${ }^{1}$ Department of Neurological Surgery, Northwestern University \\ Feinberg School of Medicine, Chicago, Illinois, USA; \\ 2Department of Biochemistry and Molecular Genetics, Northwestern \\ University Feinberg School of Medicine, Chicago, Illinois, USA
}

\begin{abstract}
Diffuse intrinsic pontine glioma (DIPG) is a rare but uniformly fatal cancer of the brain, with peak incidence in children of 5-7 years of age. In contrast to most types of human cancer, there has been no significant improvement in treatment outcomes for patients with DIPG. Since DIPG occurs in the brainstem, a vital region of the brain, there are no surgical options for providing relief to patients, and chemotherapy as well as radiation therapy provide palliative relief at best. To date, more than 250 clinical trials evaluating radiotherapy along with conventional cytotoxic chemotherapy, as well as newer biologic agents, have failed to improve the dismal outcome when compared with palliative radiation alone. The recent discovery of somatic oncogenic histone gene mutations affecting chromatin regulation in DIPG has dramatically improved our understanding of the disease pathogenesis in DIPG, and these findings have stimulated the development of novel therapeutic approaches targeting epigenetic regulators for disease treatment. This review will discuss about the role of histone modification in chromatin machinery and epigenetic therapeutic strategies for the treatment of DIPG.
\end{abstract}

Key words: histone, methylation, demethylation, pediatric brain tumor, DIPG

\section{Introduction}

Brain tumors are the main cause of cancer-related morbidity and mortality in children, representing the second-most common cancer and the most common solid tumor in childhood. Among brain tumors, Diffuse Intrinsic Pontine Gliomas (DIPGs) are the most aggressive primary brain tumors in children. ${ }^{1-3)}$ DIPG originates in the pons and is seen almost exclusively in children, with a median age at diagnosis of 6-7 years. ${ }^{4-7)}$ DIPGs represent approximately $10 \%$ of all brain tumors in children, and are typically fatal within two years after diagnosis. ${ }^{8,9)}$

DIPG remains one of the most severe challenges within pediatric neuro-oncology. The mainstay of treatment for DIPG remains radiation (54-60Gy) therapy, fractionated over a six-week period, which often provides transient symptom relief in approximately $70-80 \%$ of patients. No survival benefit has been achieved with alternative radiation strategies or a combination with radiation sensitizers. ${ }^{10,11)}$ Unfortunately, cancer recurrence and patient demise is a certainty, with

Received January 25, 2017; Accepted April 11, 2017 the median overall survival for patients with DIPG of approximately 10 months. Median progression-free survival is between 7 and 8 months, with survival after relapse measured in weeks. To date, more than 250 clinical trials, including standard and novel chemotherapeutics using a variety of regimens, along with radiation therapy, have been conducted, but none has demonstrated significant improvement in treatment outcomes for patients with DIPG. ${ }^{12)}$

Major obstacles for the development of effective treatments for DIPGs include extensive tumor cell infiltration at the time of diagnosis, an eloquent anatomic location that is inoperable, and poor response to radiotherapy. In the past 20 years, there has been minimal improvement in DIPG outcomes. ${ }^{13,14}$ A fundamental limitation in the development of biologically targeted therapies for DIPGs is that the diagnosis is usually confirmed by imaging studies, and surgical biopsy is rarely performed due to associated risk. ${ }^{15)}$ The lack of tissue samples, together with a limited understanding of DIPG tumor biology, has significantly hindered progress in identifying underlying oncogenic steps that contribute to the development of DIPG. 
However, recent advances in stereotactic neurosurgery have enabled surgeons to obtain reliable biopsy tissue for histological and genomic analysis, and as well for the establishment of primary tumor cells and patient-derived xenograft (PDX) models, with a low morbidity of less than $4 \%$ of patients. ${ }^{16-19)}$ Given the relative safety of stereotactic biopsy, sufficient numbers of DIPGs were eventually collected to enable the identification of recurrent gene alterations, the most common of which results in the substitution of methionine for lysine at position 27 of histone $\mathrm{H} 3$ variants (to be referred to as $\mathrm{K} 27 \mathrm{M}){ }^{20,21)}$ Despite being just one of 32 genes that encode histone $\mathrm{H} 3$ peptides in a diploid cell, this mutation causes a remarkable hypomethylation of a significant fraction of all wild-type histone H3K27 in cells bearing the K27M mutation. ${ }^{2-26)} \mathrm{K} 27 \mathrm{M}$ mutant DIPG is associated with a more aggressive clinical course with poor overall response to therapy. ${ }^{27)}$ The molecular as well as tumor biological consequences of, and therapeutic options for treating K27M DIPG are subjects of intense interest, and the current research described here is intended to advance our understanding of these aspects of this intriguing gene alteration.

\section{Histone modification and chromatin machinery}

Identification of the role of histone modification and the functional involvement of chromatin machinery is necessary to understand cancer biology, and to develop effective therapeutic strategies in human cancer. Histones are the major protein component of eukaryotic chromatin and are deposited during DNA synthesis. DNA is packaged by eight histone proteins, and this octamer regulates transcription mediated by selective and reversible modifications of nucleosomal $\mathrm{DNA}^{28,29)}$ and histone tails.

Histone modifications are known to control the transition in conformation between genes that are active transcriptionally and those that are inactive, and function as molecular switches, suppressing or activating gene expression or altering the expression levels of genes. ${ }^{30-32)}$ Histone N-terminal tails are subject to post-translational modification, including lysine and arginine methylation, lysine acetylation, serine and threonine phosphorylation, and lysine ubiquitination or sumoylation. ${ }^{32-34)}$ These modifications are catalyzed by enzymes that are called "writers," which include histone methyltransferases, or "erasers," including histone demethylase and histone deacetylases (HDACs). There are also proteins called "readers" that are associated with chromatin and are involved in recruiting additional proteins with chromatin-modifying functions. ${ }^{35-37)}$

\section{Histone acetylation}

Acetylation of histones increases a negative charge to the protein, loosening the interaction of negatively charged DNA with the histone, leading to active and open chromatin. Histone acetyltransferases (HATs) as writers and HDACs as erasers antagonistically control histone acetylation. Acetylation is considered a histone mark for transcriptional activation, so HATs are associated with active genes, while HDACs are associated with inactive genes. A recent genome-wide mapping of HAT and HDAC binding on chromatin revealed that both are found at active genes with acetylated histones ${ }^{38)}$ and are targeted to transcribed regions of active genes by phosphorylated RNA Pol II. The majority of HDACs in the human genome function to reset chromatin by removing acetylation at active genes. ${ }^{38)}$

Acetylated histones recruit specific chromatinassociated bromodomain and extraterminal (BET) proteins. ${ }^{35,39)}$ The BET protein bromodomain-containing 4 (BRD 4) is a lysine acetylation "reader", and plays a role in initial recognition of acetylated histones. The next step is "mediator" recruitment, which is a transcription initiation cofactor, to promoters, which leads to RNA Pol II phosphorylation. Recently, BRD4 was identified as playing a possibly important role in brain cancer development. ${ }^{40-43)}$

\section{Histone methylation}

In contrast to histone acetylation, methylation of histones does not change the charge of histones. Rather, a docking site is created for chromatin-associated proteins containing specific methyl histone-binding domains. Lysine residues of histones can be mono-, di-, or tri-methylated, and each of these modifications has a specific biologic effect. Transcriptionally active euchromatin, for example, can be found associated with methylation of H3K4, H3K36, and H3K79. Alternatively, methylation of H3K9, H3K27, and H4K20 typically can be found in association with transcriptionally repressed heterochromatin. Of the well characterized modifications, H3K4me3 is associated primarily with active promoters, ${ }^{44,45}$ while H3K27me3 correlates with silencing by the polycomb repressive complex 2 (PRC2). PRC2 is composed of the SET domain-containing histone methyltransferase EZH2 (enhancer of zeste homolog 2) or its functional homologue EZH1, and histone core accessory proteins (EED, SUZ12, and RbAp48), and the PRC2-associated factors JARID2 and ASXL1 ${ }^{46-48)}$ (Fig. 1). The H3K27me3 mark is recognized by the PRC1 complex, which represses transcription by several mechanisms, including ubiquitination of histone H2A on lysine K119 and chromatin compaction. 


\section{EUCHROMATIN}
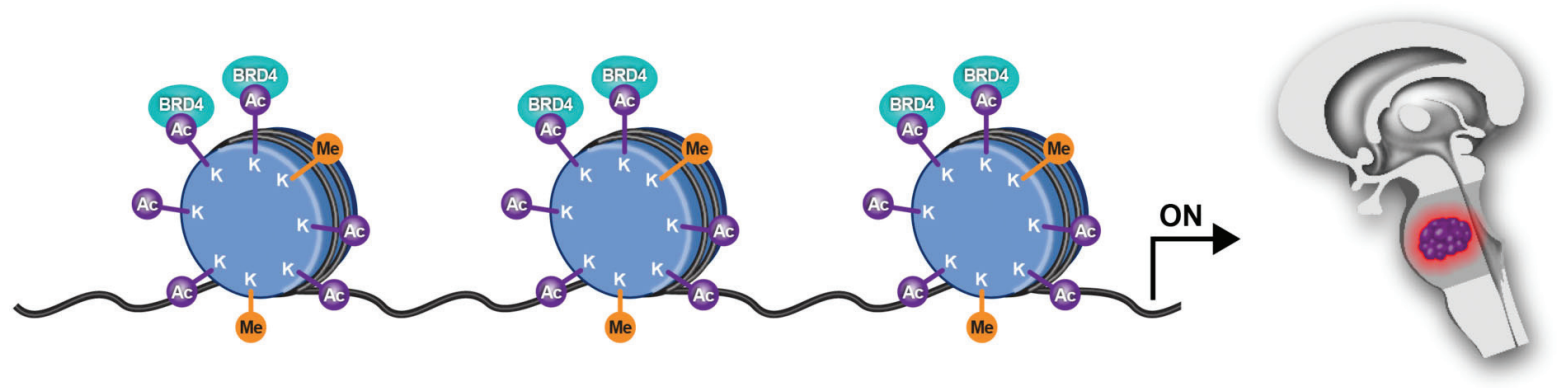

PCR2

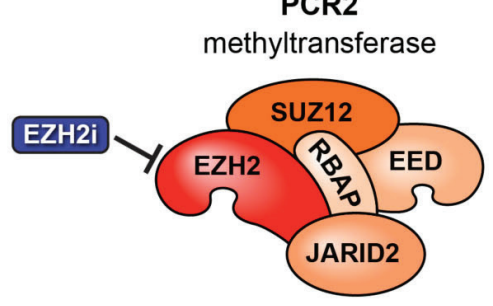

KDM6

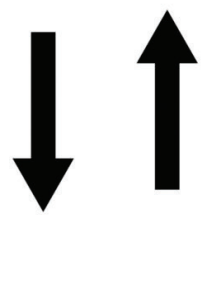

demethylase

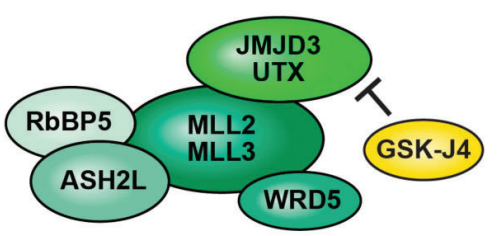

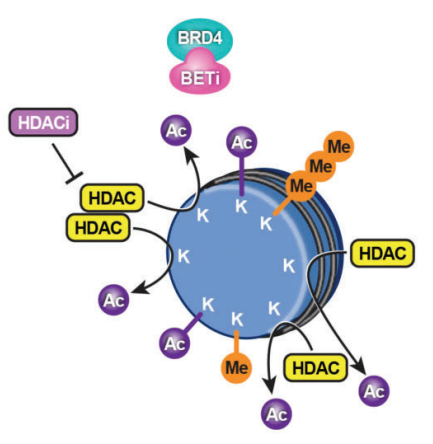

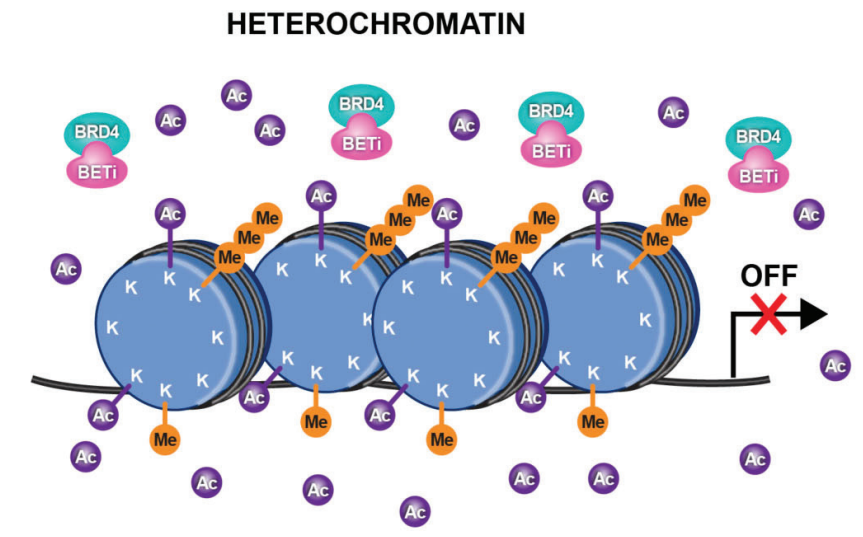

Methylation

Acetylation

EZH2i

$E Z H 2$ inhibitor

HDACI HDAC inhibitor

\section{BETi BET bromodomain inhibitor}

Fig. 1 New epigenetic therapies for DIPGs. In DIPG, H3K27M mutations (histone H3.1 or H3.3) lead to hypomethylation of $\mathrm{H3K27}$, which promotes a more accessible chromatin state characterized by $\mathrm{H} 3 \mathrm{~K} 27$ acetylation and aberrant gene expression (upper: Euchromatin). H3К27M mutations inhibits the major H3K27 methylase PRC2. Treatment of DIPG with K27 demethylase inhibitor GSKJ4 results in increased K27me2 and K27me3 and, reduced tumor growth (lower: Heterochromatin). Moreover, treatment with the non-specific HDAC inhibitor panobinostat demonstrated an increase in global H3 acetylation increasing/restoring H3K27me3 levels. In addition, competitive binding with BET bromodomain inhibitors prevents the interaction of BRD4 with acetylated histone, leading to the repression of BRD4 transcriptional targets and also limiting tumor growth. Reprinted by permission from Taylor \& Francis Group, LLC: Epigenetics [January 6, Epub ahead of print], copyright 2017. 
Histone lysine demethylases (KDMs), which remove methyl group(s) from lysine side chains (Fig. 1), also provide dynamic control of histone methylation. Methyltransferase and demethylases, with their opposing functions, strive to maintain balanced levels of histone methylation. H3K27 methylation is regulated by demethylase, including that of ubiquitously transcribed tetratricopeptide repeat, $\mathrm{X}$ chromosome (UTX)/KDM6A and jumonji domain containing-3 (JMJD3)/KDM6B. ${ }^{49-56)}$ UTX and JMJD3 both function as components of a transcriptional activator complex with the MLL2/MLL3 (mixedlineage leukemia protein 2/3) H3K4 methyltransferases, suggesting dual roles of these enzymes in both removing H3K27 methyl marks and adding methyl groups to H3K4.48)

The genes encoding KDM5A (JARID1A); KDM5C (JARID1C) have been found to have mutations, affecting H3K4 methylation and as has KDM6A (UTX), which affects H3K27 methylation. ${ }^{57-60)}$ The activity of enzymes controlling H3K27 methylation has been found to be both increased and decreased in other cancers, ${ }^{48,61)}$ suggesting that a methylation balance is critical for normal cell growth and for a context-dependent role of polycomb proteins and KDMs in malignancy.

\section{DNA methylation}

There have been detailed investigations of DNA methylations in adult GBM, which aided the development of clinical trials, as these relate to tumor recurrence and radiotherapy resistance. By applying genome-wide DNA methylation profiling in pediatric and adult patient cohorts, recurrent age-specific mutations in H3F3A were observed, while tumors with frequent PDGFRA alterations were found in patients wider age range. ${ }^{62)}$ The same approach helped in subgrouping DIPG patients on the basis of CpG island methylation, and identified a subgroup with high-level amplification of MYCN and high-grade histology ${ }^{63)}$ Observations of the DNA methylation pattern were associated with changes in a specific histone 3 variant mark. ${ }^{19)}$ Recent observation of inactivation of SET-domain-containing histone methyltransferases by K27M variants, ${ }^{23)}$ which may explain the specific DNA methylation pattern in H3.3K27M non-brainstem tumors. ${ }^{61)}$ Since DNA methylation profiles are associated with the K27M mutation irrespective of tumor location, this suggests that the K27M mutation has a role in driving the epigenetic phenotype. Moreover, gain-of-function mutations in ACVR1, FGFR1, and PDGFRA also were found to be associated with H3K27M variants. ${ }^{19}$

\section{Histone gene mutations in DIPG}

Recent exome sequencing studies of pediatric high-grade gliomas have identified gain-of-function mutations in $\mathrm{H} 3$ histone genes: histone $3 \mathrm{~A}$ ( $\mathrm{H} 3 \mathrm{~F} 3 \mathrm{~A})$ and histone H3b (HIST1H3B), encoding histone H3 variants H3.3 and H3.1 respectively. There are two specific residues that are mutated in histone H3.3, lysine at position 27 for methionine (K27M) or glycine at position $34^{56)}$ for valine or arginine (G34V/R). K27M is observed in approximately $80 \%$ of DIPGs, ${ }^{20,21)}$ mostly involving $H 3 F 3 A$, with the rest involving HIST1H3B. ${ }^{21,27,62-65)} \mathrm{G} 34 \mathrm{~V} / \mathrm{R}$ mutations, however, are observed only in H3F3A.

Since it occurs frequently and confers a poor prognosis, the role of the histone H3K27M mutation has been studied extensively in tumor initiation, maintenance and progression. ${ }^{66,67)}$ Lewis and others have identified a gain-of-function mechanism for this mutation: the $\mathrm{K} 27 \mathrm{M}$ mutant protein sequesters PRC2, which normally represses gene expression through histone methylation, and functionally inactivates it. ${ }^{22-25}$ Suppression of PRC2 by K27M leads to a dramatic reduction of methylation of wild-type H3K27, greatly in excess of the proportion of K27M-to-wild type H3, leading to extensive transcriptional reprogramming of tumor cells. ${ }^{22,24)}$ The effect of K27M on PRC2 function and wild type K27 methylation results in both increased and decreased gene expression. While there is a largescale decrease in total chromatin $\mathrm{H} 3$ di-methylation (K27me2) and tri-methylation (K27me3), and the associated activation of transcription as a resulted of decreased K27 methylation, specific genes are repressed with gain of H3K27 methylation in DIPG. $\mathrm{K} 27 \mathrm{M}$ is associated, therefore, with both activation and repression of gene expression. The identification of the specific genes activated and repressed remains are a subject of intensive investigation, with some findings being recently made. Using ChIP-seq and whole-genome sequencing in high-grade gliomas in children, Bender et al. found that DNA hypomethylation and reduced H3K27me3 levels work together to induce gene expression in high-grade gliomas bearing K27M mutations. ${ }^{24)}$ However, Chang et al. found in $\mathrm{H} 3 \mathrm{~K} 27 \mathrm{M}$ mutant tumors that the genes with increased H3K27me3 were associated with cancer development pathways. ${ }^{22}$

It is of interest, and potential significance, that recent study from Drosophila melanogaster constitutively expressing K27M demonstrated that H3K27 acetylation (H3K27ac) levels and associated bromodomaincontaining proteins (BRD 1 and 4) are increased in K27M-containing nucleosomes. ${ }^{38)}$ The Drosophila K27M mutant animal models resemble PRC2 lossof-function phenotypes, causing reduction of H3K27 methylation and derepression of PRC2 target genes, that may indicate similar molecular pathogenesis of $\mathrm{K} 27 \mathrm{M}$ pediatric glioma models. Other histone 
lysine-to-methionine mutations (i.e., H3K9M) have been tested in Drosophila, consistent with H3K27M mutation, showing reduced methylation levels and a possible role in heterochromatic silencing. ${ }^{40)}$

In a most recent study of patient-derived K27M DIPG models, Piunti et al. found that K27M mutant associates with increased H3K27ac and the heterotypic H3K27M-K27ac nucleosomes colocalize with bromodomain proteins at the loci of actively transcribed genes, whereas PRC2 is excluded from these regions, suggesting that H3K27M does not sequester PRC2 activity. ${ }^{43}$

To further understand the role of the H3.3K27M mutation in DIPG tumorigenesis, Funato et al. created a mouse model of DIPG by transducing the gene encoding H3.3K27M into neural progenitors derived from human embryonic stem (ES) cells. ${ }^{68)}$ The oncogenic transformation in neural progenitors was promoted by a synergy between genetic modifications of H3.3K27M, p53 loss, and PDGFRA activation in these particular cells. Importantly, the K27M mutation only transformed neural progenitors derived from ES cells, and not astrocytes derived from these cells or the ES cells themselves. These findings suggest the specificity of K27M-associated transformation for a specific cellular context. H3.3K27M expression also upregulated stem cell-associated genes such as LIN28B, PLAG1, and PLAGL1, while suppression of the same genes reduced growth of these tumor cells. ${ }^{68)}$

In another study regarding the oncogenic function of H3.3K27M, immortalized human astrocytes were transfected with N-terminally flag-tagged H3.3 wild type (WT) and H3.3K27M, and alterations in both expression and methylation profiles of the H3.3K27M vs. WT expressing cells were observed. ${ }^{63)}$ Molecular and cellular functions, particularly increased cell-tocell signaling and decreased cell cycle progression and proliferation, were the top pathways altered by H3.3K27M expression. H3.3K27M-expressing cells showed global reduction of H3K27me3 compared with H3.3WT cells. Likewise, reduced H3K27me3 levels relative to WT tumors were observed by immunohistochemical staining of patient-derived K27M DIPGs. However, expression of H3K4me3 and H3K9ac did not change with these mutations. ${ }^{63)}$ These investigations, taken together, reveal the impact of $\mathrm{K} 27 \mathrm{M}$ mutations on methylation patterns, gene expression, and transformation.

Histone gene mutations in DIPG are also associated with further genetic alterations. ${ }^{65,69,70)}$ Mutations in activin receptor type 1 (ACVR1, also known as ALK2 (activin receptor-like kinase-2)) are observed frequently $(80 \%)$ in DIPG with histone H3.1 K27M mutation, but do not occur in other gliomas. ${ }^{17,19,65,71)}$
ACVR1 mutations constitutively activate BMPdependent transforming growth factor (TGF)- $\beta$ signaling, with consequent SMAD phosphorylation, leading to increased SMAD target gene transcription. Histone mutations are also associated with oligodendrocyte lineage transcription factors 1 and 2 (OLIG1 and OLIG2) expression, as well as with a forebrain marker, forkhead box G1 transcription factor (FOXG1). High OLIG1 and OLIG2 and low FOXG1 are observed in H3.3 K27M tumors.

\section{Epigenetic targeted therapy for DIPG}

Altered epigenetics, either alone or in combination with gene mutations, can play an important role in tumor initiation and progression. The possibility to revert epigenetic changes has recently proven valuable to developing an epigenetic targeted therapy for pediatric brain tumors. ${ }^{26,27,35-37,72-77)}$ Drugs targeting epigenetic modifiers, including the inhibitors of histone methyltransferases, demethylases, HDACs and BET proteins, have emerged recently in clinical trials for these tumors (Table 1).

\section{Targeting histone methylation}

Because global reduction of H3K27 methylation is a key epigenetic event in K27M DIPG, pharmacologic restoration of H3K27 methylation is a rational therapeutic strategy for this lethal pediatric malignancy (Fig. 1). In theory, there are two pharmacological paths to restoring global H3K27 methylation in DIPG: either enhancing PRC2 methyltransferase activity or inhibiting demethylase activity for the lysine 27 residue. Histone H3K27 is methylated by EZH2, a component of PRC2, and is demethylated by the K27 demethylases JMJD3 and UTX. ${ }^{48-56)}$ Recently, a nanomolar inhibitor against JMJD3, GSKJ4, was shown to increase cellular H3K27 methylation in association with its potential use to treat immune disorders ${ }^{78)}$ (Fig. 1). GSKJ4 is considered a prodrug, and its ethyl ester derivative, GSKJ1, is the active histone demethylase inhibitor, and upon hydrolysis GSKJ4 is converted to GSKJ1 in vivo. GSKJ4 has recently been used successfully to treat brainstem gliomas in vitro and in vivo ${ }^{26,71,79-81)}$ (Fig. 1). GSKJ4 treatment promotes increased $\mathrm{K} 27 \mathrm{me} 2$ and $\mathrm{K} 27 \mathrm{me} 3$ in K27M-mutant DIPG cells, as well as in human astrocytes modified to express K27M. ${ }^{26)}$ GSKJ4 treatment-associated increases in K27me2 and K27me3 are, in turn, associated with a marked dose- and time-dependent inhibition of K27M mutant DIPG cell growth. In contrast, GSKJ4 has little effect against pediatric glioma cells with wild type and G34V mutant $H 3 F 3 A$. In addition to its anti-proliferative activity, GSKJ4 increased the apoptosis of K27M mutant cells, while having no significant effect on 


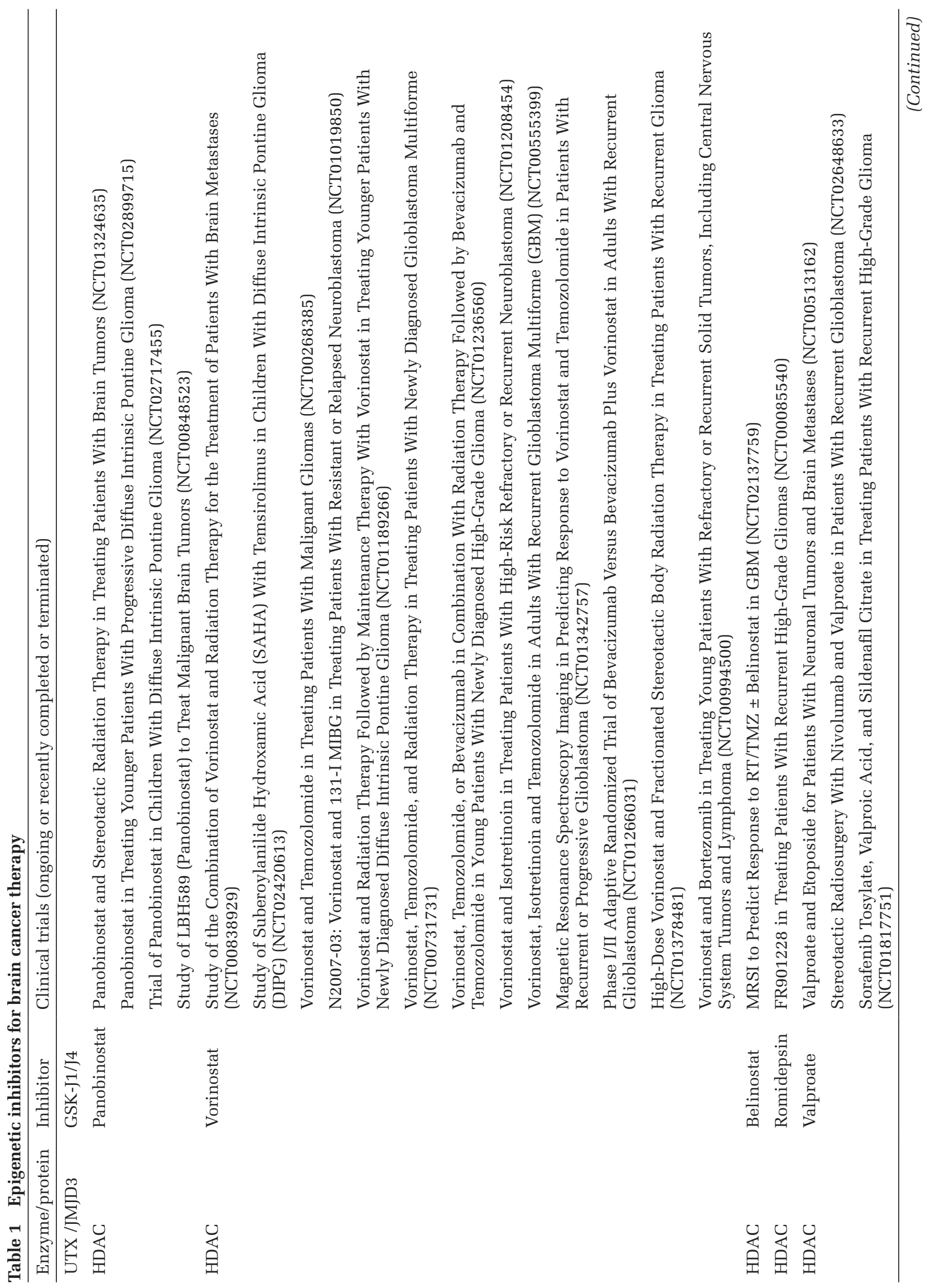


apoptosis for cells expressing wild-type or the G34V mutant. Importantly, pharmacologic inhibition of JMJD3 in DIPG orthotopic xenografts reduced tumor growth and significantly extended animal survival but did not achieve the same effect in animals with xenografts containing wild-type H3.3. These results suggested that GSKJ4 anti-tumor activity is specific to $\mathrm{K} 27 \mathrm{M}$ mutant tumors, and this $\mathrm{K} 27 \mathrm{M}$ anti-tumor activity is associated with increased $\mathrm{K} 27 \mathrm{me} 2$ and K27me3. The penetration of GSKJ4 into the brain, including the brainstem where DIPG tumors develop, was assessed by high-performance liquid chromatography following systemic administration of the drug, and suggesting that GSKJ4 be explored as a potential targeted therapy for patients with DIPG. ${ }^{26)}$

Of interest, GSKJ4 showed a potential therapeutic activity for T-cell acute lymphoblastic leukemia (T-ALL), a hematologic malignancy driven by NOTCH1 signaling, loss-of-function mutations and deletion of the genes EZH2 and SUZ12; JMJD3 is expressed at higher levels in T-ALL relative to other leukemias. ${ }^{58,82,83)}$ GSKJ4 reduced cell proliferation and increased $\mathrm{K} 27 \mathrm{M}$ methylation in T-ALL. ${ }^{58)}$ The main mechanism of GSKJ4 in T-ALL appears to be through JMJD3 inhibition. ${ }^{58)}$

For both T-ALL and DIPG, inhibition of UTX, the other known H3K27 demethylase, did not reduce proliferation of tumor cells. Furthermore, in T-ALL, the genes suppressed by GSKJ4 had significant overlap with genes upregulated by UTX knocking down. Taken together, these results point to distinct roles in chromatin modification for UTX and JMJD3, and suggest that JMJD3 is a likely therapeutic epigenetic target for treating pediatric cancers.

Besides increasing H3K27 methylation by GSKJ4mediated inhibition of the JMJD3 demethylase, a recent study of patient-derived DIPG models demonstrated that inhibition of residual EZH2 activity decrease H3K27 methylation at the promoter of tumorsuppressor protein p16INK4a (encoded by CDKN2A (cyclin- dependent kinase inhibitor 2A)) and reduces cell proliferation, implicating that EZH2 inhibition presents an epigenetic therapeutic strategy for DIPG. ${ }^{84)}$

\section{Targeting histone acetylation}

The elevated level of H3K27 acetylation in DIPG has also been a potential epigenetic target by treating with HDAC inhibitors (Table 1). HDAC inhibitors increase histone acetylation by inhibiting histone deacetylation, leading to an open chromatin structure and gene activation (Fig. 1). A non-selective FDA-approved potent HDAC inhibitor, panobinostat (LBH589, Farydak), has been developed for the treatment of various cancers ${ }^{85}$ (Table 1). A recent chemical screening in patient-derived DIPG cells 
identified panobinostat as having potent anti-tumor activity against DIPG in vitro. ${ }^{86)}$ In fact, panobinostat induced a dose-dependent increase in global H3 acetylation as well as H3K27 methylation, and reduced the expression of oncogenes (e.g., MK167, CCND1). Polyacetylation of the H3 N-terminal tail by panobinostat can 'detoxify' K27M-induced inhibition of PRC2 and rescue the H3K27 hypomethylation phenotype. Synergy between panobinostat and GSKJ4 was observed in DIPG cells, ${ }^{86}$ highlighting the importance of investigating the anti-tumor activities resulting from simultaneous inhibition of multiple histone modifier.

In a recent study in genetic and orthotopic DIPG models, Hennika et al. found, consistent with previous findings, ${ }^{86)}$ that panobinostat demonstrated significant anti-tumor activity in vitro and in short-term in vivo efficacy studies. ${ }^{87)}$ However, the efficacy of panosbinostat was unrelated to $\mathrm{H} 3$ status in this study. Moreover, panobinostat treatment at its well-tolerated dose did not increase overall survival in the K27M DIPG models, suggesting that substantial toxicity would arise in using a panobinostat concentration and treatment duration sufficient to provide an overall survival benefit. Given the important functions of histone acetylation in normal physiology, HDAC inhibition could cause off-target effects with systemic administration. The accessibility at the appropriate central nervous system (CNS) location of agents targeting epigenetic modifiers should be assessed in well-designed toxicological studies. The challenge for HDAC inhibitors will be to achieve effective concentrations able to inhibit the target, which would require either direct CNS administration or sufficient penetration of the blood-brain barrier with systemic administration, with a large enough therapeutic window and an acceptable toxicity profile, considering the ongoing normal development of pediatric patients. To define the adverse effect and maximum tolerated dose, phase I clinical trials of this compound are currently being tested in children with DIPG (NCT02717455, NCT02899715). NCT02717455 is sponsored phase 1 clinical trial of panobinostat in children with recurrent and progressive DIPG by Pediatric Brain Tumor Consortium (PBTC) and currently recruiting the patient with age from 2 to 21 year olds. Panobinostat will be administered orally every other day, 3 times a week for three weeks, followed by one week off of therapy. Three weeks of therapy plus the one-week rest period (total 4 weeks) will constitute one course. Treatment will continue for up to 26 courses (approximately 2 years) barring progressive disease or unacceptable toxicity. NCT02899715 is sponsored phase 1 clinical trial of panobinostat in children with DIPG by National Cancer Institute
(NCI) and currently recruiting the patient. Patients will receive panobinostat orally thrice weekly for 3 weeks. Treatment repeats every 28 days for 26 courses ( 2 years) in the absence of disease progression or unacceptable toxicity. In addition to the pharmacokinetic (PK) analysis of panobinostat, this trial will measure changes of H3K27M mutations in cell-free DNA from peripheral blood and urine samples.

\section{Targeting BET proteins}

In addition to pharmacologic inhibition of histone acetylation by HDAC inhibitors, highly selective BET protein inhibitors, such as JQ-1, ${ }^{39,43,88,89)}$ I-BET151, ${ }^{90,91)}$ I-BET762, ${ }^{92)}$ INCB054329, ${ }^{42)}$ and OTX-015, ${ }^{93)}$ have been generated to target specifically the recognition of acetylated lysine residues (Fig. 1) (Table 1). The BET bromodomain BRD4 binds to acetylated histones through its conserved bromodomains and activates transcription. ${ }^{74)}$ Competitive binding of BET inhibitors to the bromodomain pocket results in displacement of BRD4 from acetylated histones in active chromatin, leading to transcriptional inactivation (Fig. 1). ${ }^{74,88,89,91,94,95)}$ Several preclinical studies with the BET inhibitors JQ1 and I-BET151 have been conducted in glioma models including DIPG, with encouraging antitumor activity. ${ }^{43,96,97)}$ Moreover, a novel BET inhibitor, OTX-015 (MK-8628), was tested at the clinical level in dose-finding studies in glioblastoma (GBM) patients (NCT02296476), having shown antitumor effects in vitro and in vivo as monotherapy and in combination with conventional treatment in GBM models ${ }^{42)}$ (Table 1). A phase $1 / 2$ study of another BET inhibitor, INCB057643, is also currently being tested in advanced solid tumors (NCT02711137) (Table 1). Of note, the anti-proliferative effects of BET inhibitors in GBM may be mediated, at least in part, by reduced expression of the long non-coding RNA HOX transcript antisense RNA (HOTAIR), which has tumor-promoting effects. ${ }^{98)}$

\section{Conclusion}

Recently, our knowledge of DIPG genetics and epigenetics has significantly improved in association with the discovery of histone gene mutations. Current investigations are underway to define the function of histone mutations and to determine how they interact with other known oncogenes. Further investigation is needed to understand the mechanisms of action of the mutations observed DIPG and the corresponding alterations in chromatin machinery, in addition to their complex interactions with other known oncogenes. Therapeutic targeting of histone modifiers responsible for chromatin formation and epigenetic regulation of gene expression is a rela- 
tively new strategy and an area of increased interest in pediatric neuro-oncology. Ongoing clinical trials are evaluating the anti-tumor activity of histone modifier inhibitors, and also any interactions with conventional genotoxic therapies, with the goal of improving outcomes for children with DIPG. Given the important functions of histone modifications in normal physiology, optimizing the dose and frequency of administration of inhibitors of histone modifiers to maximize the therapeutic window and limit toxicity will be a key factor for reaching a successful clinical outcome. The combination of agents with different mechanisms of action may offer a better chance for tumor control with limited toxicity and a reduced likelihood of acquired resistance compared with single agent therapy. Clinical trials should include approaches for monitoring tumor treatment response, which will need the identification and validation of non-invasive biomarkers for residual tumors, and for assessing the molecular effects of treatment through analysis of tumor tissue or other tissue samples obtained during and/or after treatment.

\section{Acknowledgment}

R.H. was supported by US National Institutes of Health (NIH) grant NS093079, and the Bear Necessities Pediatric Cancer Foundation and Rally Foundation, and the John McNicholas Pediatric Brain Tumor Foundation. We thank Michael Gallagher for illustration of the figure and Accixx Biomedical Consulting (www.Accixx.com) for editorial assistance.

\section{Conflicts of Interest Disclosure}

The author declares that there is no conflict of interest with the content of this report.

\section{References}

1) Wong ET, Hess KR, Gleason MJ, et al.: Outcomes and prognostic factors in recurrent glioma patients enrolled onto phase II clinical trials. J Clin Oncol 17: 2572-2578, 1999

2) Buckner JC: Factors influencing survival in high-grade gliomas. Semin Oncol 30: 10-14, 2003

3) Louis DN, Ohgaki H, Wiestler OD, et al.: The 2007 WHO classification of tumours of the central nervous system. Acta Neuropathol 114: 97-109, 2007

4) Freeman CR, Farmer JP: Pediatric brain stem gliomas: a review. Int J Radiat Oncol Biol Phys 40: 265-271, 1998

5) Berger MS, Edwards MS, LaMasters D, Davis RL, Wilson CB: Pediatric brain stem tumors: radiographic, pathological, and clinical correlations. Neurosurgery 12: 298-302, 1983
6) Littman P, Jarrett P, Bilaniuk LT, et al.: Pediatric brain stem gliomas. Cancer 45: 2787-2792, 1980

7) Hargrave D, Bartels U, Bouffet E: Diffuse brainstem glioma in children: critical review of clinical trials. Lancet Oncol 7: 241-248, 2006

8) Donaldson SS, Laningham F, Fisher PG: Advances toward an understanding of brainstem gliomas. J Clin Oncol 24: 1266-1272, 2006

9) Frazier JL, Lee J, Thomale UW, Noggle JC, Cohen KJ, Jallo GI: Treatment of diffuse intrinsic brainstem gliomas: failed approaches and future strategies. J Neurosurg Pediatr 3: 259-269, 2009

10) Robison NJ, Kieran MW: Diffuse intrinsic pontine glioma: a reassessment. J Neurooncol 119: 7-15, 2014

11) Roos DE, Smith JG: Randomized trial on radiotherapy for paediatric diffuse intrinsic pontine glioma (DIPG). Radiother Oncol 113: 425, 2014

12) Warren KE: Diffuse intrinsic pontine glioma: poised for progress. Front Oncol 2: 205, 2012

13) Fangusaro J: Pediatric high-grade gliomas and diffuse intrinsic pontine gliomas. J Child Neurol 24: 1409-1417, 2009

14) Kebudi R, Cakir FB: Management of diffuse pontine gliomas in children: recent developments. Paediatr Drugs 15: 351-362, 2013

15) Barkovich AJ, Krischer J, Kun LE, et al.: Brain stem gliomas: a classification system based on magnetic resonance imaging. Pediatr Neurosurg 16: 73-83, 1990

16) Roujeau T, Machado G, Garnett MR, et al.: Stereotactic biopsy of diffuse pontine lesions in children. J Neurosurg 107: 1-4, 2007

17) Puget S, Philippe C, Bax DA, et al.: Mesenchymal transition and PDGFRA amplification/mutation are key distinct oncogenic events in pediatric diffuse intrinsic pontine gliomas. PLoS One 7: e30313, 2012

18) Geoerger B, Hargrave D, Thomas F, et al.: ITCC (Innovative Therapies for Children with Cancer) European Consortium: Innovative Therapies for Children with Cancer pediatric phase I study of erlotinib in brainstem glioma and relapsing/refractory brain tumors. Neuro-oncology 13: 109-118, 2011

19) Fontebasso AM, Papillon-Cavanagh S, Schwartzentruber J, et al.: Recurrent somatic mutations in ACVR1 in pediatric midline high-grade astrocytoma. Nat Genet 46: 462-466, 2014

20) Schwartzentruber J, Korshunov A, Liu XY, et al.: Driver mutations in histone H3.3 and chromatin remodelling genes in paediatric glioblastoma. Nature 482: 226-231, 2012

21) Wu G, Broniscer A, McEachron TA, et al.: St. Jude Children's Research Hospital-Washington University Pediatric Cancer Genome Project: Somatic histone $\mathrm{H} 3$ alterations in pediatric diffuse intrinsic pontine gliomas and non-brainstem glioblastomas. Nat Genet 44: 251-253, 2012

22) Chan KM, Fang D, Gan H, et al.: The histone H3.3K27M mutation in pediatric glioma reprograms H3K27 methylation and gene expression. Genes Dev 27: 985-990, 2013 
23) Lewis PW, Müller MM, Koletsky MS, et al.: Inhibition of PRC2 activity by a gain-of-function H3 mutation found in pediatric glioblastoma. Science 340: 857-861, 2013

24) Bender S, Tang Y, Lindroth AM, et al.: Reduced H3K27me3 and DNA hypomethylation are major drivers of gene expression in $\mathrm{K} 27 \mathrm{M}$ mutant pediatric high-grade gliomas. Cancer Cell 24: 660-672, 2013

25) Venneti S, Garimella MT, Sullivan LM, et al.: Evaluation of histone 3 lysine 27 trimethylation (H3K27me3) and enhancer of Zest 2 (EZH2) in pediatric glial and glioneuronal tumors shows decreased H3K27me3 in H3F3A K27M mutant glioblastomas. Brain Pathol 23: 558-564, 2013

26) Hashizume R, Andor N, Ihara Y, et al.: Pharmacologic inhibition of histone demethylation as a therapy for pediatric brainstem glioma. Nat Med 20: 1394-1396, 2014

27) Khuong-Quang DA, Buczkowicz P, Rakopoulos P, et al.: K27M mutation in histone H3.3 defines clinically and biologically distinct subgroups of pediatric diffuse intrinsic pontine gliomas. Acta Neuropathol 124: 439-447, 2012

28) Churchman LS, Weissman JS: Nascent transcript sequencing visualizes transcription at nucleotide resolution. Nature 469: 368-373, 2011

29) Hodges C, Bintu L, Lubkowska L, Kashlev M, Bustamante C: Nucleosomal fluctuations govern the transcription dynamics of RNA polymerase II. Science 325: 626-628, 2009

30) Furey TS, Sethupathy P: Genetics. Genetics driving epigenetics. Science 342: 705-706, 2013

31) Strahl BD, Allis CD: The language of covalent histone modifications. Nature 403: 41-45, 2000

32) Henikoff S: Nucleosome destabilization in the epigenetic regulation of gene expression. Nat Rev Genet 9: 15-26, 2008

33) Rivera CM, Ren B: Mapping human epigenomes. Cell 155: 39-55, 2013

34) Bird A: Perceptions of epigenetics. Nature 447: 396-398, 2007

35) Kaelin WG, McKnight SL: Influence of metabolism on epigenetics and disease. Cell 153: 56-69, 2013

36) Black JC, Van Rechem C, Whetstine JR: Histone lysine methylation dynamics: establishment, regulation, and biological impact. Mol Cell 48: 491-507, 2012

37) Arcipowski KM, Martinez CA, Ntziachristos P: Histone demethylases in physiology and cancer: a tale of two enzymes, JMJD3 and UTX. Curr Opin Genet Dev 36: 59-67, 2016

38) Wang Z, Zang C, Cui K, et al.: Genome-wide mapping of HATs and HDACs reveals distinct functions in active and inactive genes. Cell 138: 1019-1031, 2009

39) Filippakopoulos P, Knapp S: Targeting bromodomains: epigenetic readers of lysine acetylation. Nat Rev Drug Discov 13: 337-356, 2014

40) Herz HM, Morgan M, Gao X, et al.: Histone H3 lysine-to-methionine mutants as a paradigm to study chromatin signaling. Science 345: 1065-1070, 2014
41) Lin CY, Erkek S, Tong Y, et al.: Active medulloblastoma enhancers reveal subgroup-specific cellular origins. Nature 530: 57-62, 2016

42) Wadhwa E, Nicolaides T: Bromodomain Inhibitor Review: Bromodomain and Extra-terminal Family Protein Inhibitors as a Potential New Therapy in Central Nervous System Tumors. Cureus 8: e620, 2016

43) Piunti A, Hashizume R, Morgan MA, et al.: Therapeutic targeting of polycomb and BET bromodomain proteins in diffuse intrinsic pontine gliomas. Nat Med 23: 493-500, 2017

44) Barski A, Cuddapah S, Cui K, et al.: High-resolution profiling of histone methylations in the human genome. Cell 129: 823-837, 2007

45) Heintzman ND, Hon GC, Hawkins RD, et al.: Histone modifications at human enhancers reflect global celltype-specific gene expression. Nature 459: 108-112, 2009

46) Müller J, Hart CM, Francis NJ, et al.: Histone methyltransferase activity of a Drosophila Polycomb group repressor complex. Cell 111: 197-208, 2002

47) Czermin B, Melfi R, McCabe D, Seitz V, Imhof A, Pirrotta V: Drosophila enhancer of Zeste/ESC complexes have a histone $\mathrm{H} 3$ methyltransferase activity that marks chromosomal Polycomb sites. Cell 111: 185-196, 2002

48) Ezponda T, Licht JD: Molecular pathways: deregulation of histone h3 lysine 27 methylation in cancerdifferent paths, same destination. Clin Cancer Res 20: 5001-5008, 2014

49) Agger K, Cloos PA, Christensen J, et al.: UTX and JMJD3 are histone H3K27 demethylases involved in HOX gene regulation and development. Nature 449: 731-734, 2007

50) Deb G, Singh AK, Gupta S: EZH2: not EZHY (easy) to deal. Mol Cancer Res 12: 639-653, 2014

51) Simon JA, Lange CA: Roles of the EZH2 histone methyltransferase in cancer epigenetics. Mutat Res 647: 21-29, 2008

52) Kleer CG, Cao Q, Varambally S, et al.: EZH2 is a marker of aggressive breast cancer and promotes neoplastic transformation of breast epithelial cells. Proc Natl Acad Sci USA 100: 11606-11611, 2003

53) Varambally S, Dhanasekaran SM, Zhou M, et al.: The polycomb group protein EZH2 is involved in progression of prostate cancer. Nature 419: 624-629, 2002

54) Hübner MR, Spector DL: Role of H3K27 demethylases Jmjd3 and UTX in transcriptional regulation. Cold Spring Harb Symp Quant Biol 75: 43-49, 2010

55) Kooistra SM, Helin K: Molecular mechanisms and potential functions of histone demethylases. Nat Rev Mol Cell Biol 13: 297-311, 2012

56) Cloos PA, Christensen J, Agger K, Helin K: Erasing the methyl mark: histone demethylases at the center of cellular differentiation and disease. Genes Dev 22: 1115-1140, 2008

57) Jones DT, Jäger N, Kool M, et al.: Dissecting the genomic complexity underlying medulloblastoma. Nature 488: 100-105, 2012 
58) Ntziachristos P, Tsirigos A, Van Vlierberghe P, et al.: Genetic inactivation of the polycomb repressive complex 2 in $\mathrm{T}$ cell acute lymphoblastic leukemia. Nat Med 18: 298-301, 2012

59) Pugh TJ, Weeraratne SD, Archer TC, et al.: Medulloblastoma exome sequencing uncovers subtypespecific somatic mutations. Nature 488: 106-110, 2012

60) Imielinski M, Berger AH, Hammerman PS, et al.: Mapping the hallmarks of lung adenocarcinoma with massively parallel sequencing. Cell 150: 1107-1120, 2012

61) Martinez-Garcia E, Licht JD: Deregulation of H3K27 methylation in cancer. Nat Genet 42: 100-101, 2010

62) Sturm D, Witt H, Hovestadt V, et al.: Hotspot mutations in H3F3A and IDH1 define distinct epigenetic and biological subgroups of glioblastoma. Cancer Cell 22: 425-437, 2012

63) Buczkowicz P, Hoeman C, Rakopoulos P, et al.: Genomic analysis of diffuse intrinsic pontine gliomas identifies three molecular subgroups and recurrent activating ACVR1 mutations. Nat Genet 46: 451-456, 2014

64) Waldmann T, Schneider R: Targeting histone modifications-epigenetics in cancer. Curr Opin Cell Biol 25: 184-189, 2013

65) Taylor KR, Mackay A, Truffaux N, et al.: Recurrent activating ACVR1 mutations in diffuse intrinsic pontine glioma. Nat Genet 46: 457-461, 2014

66) Sturm D, Bender S, Jones DT, et al.: Paediatric and adult glioblastoma: multiform (epi)genomic culprits emerge. Nat Rev Cancer 14: 92-107, 2014

67) Zadeh G, Aldape K: ACVR1 mutations and the genomic landscape of pediatric diffuse glioma. Nat Genet 46: 421-422, 2014

68) Funato K, Major T, Lewis PW, Allis CD, Tabar V: Use of human embryonic stem cells to model pediatric gliomas with H3.3K27M histone mutation. Science 346: 1529-1533, 2014

69) Bax DA, Mackay A, Little SE, et al.: A distinct spectrum of copy number aberrations in pediatric high-grade gliomas. Clin Cancer Res 16: 3368-3377, 2010

70) Paugh BS, Qu C, Jones C, et al.: Integrated molecular genetic profiling of pediatric high-grade gliomas reveals key differences with the adult disease. J Clin Oncol 28: 3061-3068, 2010

71) Becher OJ, Wechsler-Reya RJ: Cancer. For pediatric glioma, leave no histone unturned. Science 346: 1458-1459, 2014

72) Zahnow CA, Topper M, Stone M, et al.: Inhibitors of DNA methylation, histone deacetylation, and histone demethylation: a perfect combination for cancer therapy. Adv Cancer Res 130: 55-111, 2016

73) Song Y, Wu F, Wu J: Targeting histone methylation for cancer therapy: enzymes, inhibitors, biological activity and perspectives. J Hematol Oncol 9: 49, 2016

74) Popovic R, Licht JD: Emerging epigenetic targets and therapies in cancer medicine. Cancer Discov 2: 405-413, 2012

75) McGrath J, Trojer P: Targeting histone lysine methylation in cancer. Pharmacol Ther 150: 1-22, 2015
76) Liu Y, Liu K, Qin S, Xu C, Min J: Epigenetic targets and drug discovery: part 1: histone methylation. Pharmacol Ther 143: 275-294, 2014

77) Liu K, Liu Y, Lau JL, Min J: Epigenetic targets and drug discovery Part 2: Histone demethylation and DNA methylation. Pharmacol Ther 151: 121-140, 2015

78) Kruidenier L, Chung CW, Cheng Z, et al.: A selective jumonji H3K27 demethylase inhibitor modulates the proinflammatory macrophage response. Nature 488: 404-408, 2012

79) Ramaswamy V, Remke M, Taylor MD: An epigenetic therapy for diffuse intrinsic pontine gliomas. Nat Med 20: 1378-1379, 2014

80) Morales La Madrid A, Hashizume R, Kieran MW: Future Clinical Trials in DIPG: Bringing Epigenetics to the Clinic. Front Oncol 5: 148, 2015

81) Lulla RR, Saratsis AM, Hashizume R: Mutations in chromatin machinery and pediatric high-grade glioma. Sci Adv 2: e1501354, 2016

82) Van Vlierberghe P, Ambesi-Impiombato A, Perez-Garcia A, et al.: ETV6 mutations in early immature human T cell leukemias. J Exp Med 208: 2571-2579, 2011

83) Valk PJ, Verhaak RG, Beijen MA, et al.: Prognostically useful gene-expression profiles in acute myeloid leukemia. N Engl J Med 350: 1617-1628, 2004

84) Mohammad F, Weissmann S, Leblanc B, et al.: EZH2 is a potential therapeutic target for H3K27M-mutant pediatric gliomas. Nat Med 23: 483-492, 2017

85) Ellis L, Pan Y, Smyth GK, et al.: Histone deacetylase inhibitor panobinostat induces clinical responses with associated alterations in gene expression profiles in cutaneous T-cell lymphoma. Clin Cancer Res 14: 4500-4510, 2008

86) Grasso CS, Tang Y, Truffaux N, et al.: Functionally defined therapeutic targets in diffuse intrinsic pontine glioma. Nat Med 21: 555-559, 2015

87) Hennika T, Hu G, Olaciregui NG, et al.: Pre-clinical study of panobinostat in xenograft and genetically engineered murine diffuse intrinsic pontine glioma models. PLoS One 12: e0169485, 2017

88) Filippakopoulos P, Qi J, Picaud S, et al.: Selective inhibition of BET bromodomains. Nature 468: 1067-1073, 2010

89) Delmore JE, Issa GC, Lemieux ME, et al.: BET bromodomain inhibition as a therapeutic strategy to target c-Myc. Cell 146: 904-917, 2011

90) Nicodeme E, Jeffrey KL, Schaefer U, et al.: Suppression of inflammation by a synthetic histone mimic. Nature 468: 1119-1123, 2010

91) Dawson MA, Prinjha RK, Dittmann A, et al.: Inhibition of BET recruitment to chromatin as an effective treatment for MLL-fusion leukaemia. Nature 478: 529-533, 2011

92) Mirguet O, Gosmini R, Toum J, et al.: lead optimization to afford a clinical candidate inhibitor of the BET bromodomains. J Med Chem 56: 7501-7515, 2013

93) Berenguer-Daizé C, Astorgues-Xerri L, Odore E, et al.: OTX015 (MK-8628), a novel BET inhibitor, displays in vitro and in vivo antitumor effects alone and in 
combination with conventional therapies in glioblastoma models. Int J Cancer 139: 2047-2055, 2016

94) Puissant A, Frumm SM, Alexe G, et al.: Targeting MYCN in neuroblastoma by BET bromodomain inhibition. Cancer Discov 3: 308-323, 2013

95) Lovén J, Hoke HA, Lin CY, et al.: Selective inhibition of tumor oncogenes by disruption of superenhancers. Cell 153: 320-334, 2013

96) Pastori C, Daniel M, Penas C, et al.: BET bromodomain proteins are required for glioblastoma cell proliferation. Epigenetics 9: 611-620, 2014

97) Cheng Z, Gong Y, Ma Y, et al.: Inhibition of BET bromodomain targets genetically diverse glioblastoma. Clin Cancer Res 19: 1748-1759, 2013
98) Pastori C, Kapranov P, Penas C, et al.: The bromodomain protein BRD4 controls HOTAIR, a long noncoding RNA essential for glioblastoma proliferation. Proc Natl Acad Sci USA 112: 8326-8331, 2015

Address reprint requests to: Rintaro Hashizume, MD, $\mathrm{PhD}$, Department of Neurological Surgery, Biochemistry, and Molecular Genetics, Feinberg School of Medicine, Northwestern University, 300 East Superior Street, Tarry 2-709, Chicago, IL 60611, USA. e-mail: rintaro.hashizume@northwestern.edu 